\title{
Green turtle and fish identification based on acoustic target strength
}

\author{
Sunardi a,1,*, Azrul Mahfurdz ${ }^{\mathrm{b}, 2}$, Shoffan Saifullah c,3 \\ a Department of Electrical Engineering, Universitas Ahmad Dahlan, Yogyakarta 55164, Indonesia \\ b Department of Electrical Engineering, Sultan Ahmad Shah Polytechnic, Pahang 25350, Malaysia \\ c Master of Informatics Engineering, Universitas Ahmad Dahlan, Yogyakarta 55164, Indonesia \\ ${ }^{1}$ sunargm@yahoo.com; ${ }^{2}$ azrulpmu@yahoo.com.my; ${ }^{3}$ shoffan_s@yahoo.com \\ * corresponding author
}

\section{ARTICLE INFO}

\section{Article history}

Received January 15, 2018

Revised March 9, 2018

Accepted March 19, 2018

\section{Keywords}

Target strength (TS)

Green turtle

Echo power

Fast fourier transform (FFT)

Acoustic measurement

\section{ABSTRACT}

Fisherman accidentally caught sea turtles in their fishnet. It could be dangerous for its population. This study measures the turtle target strength (TS) using modified echosounder. The result could be used to improve the efficiency of turtle repellent device. The experiment conducted in a hatchery fiber tank contained saline water. The Green were 1, 3, 12 and 18 years old. This study used three species of fish, which serves to distinguish the value between fish and sea turtles. TS of the animals were calculated incorporating reference targets (sphere). The echo power of the turtle was compared with the solid steel sphere which is confirmed good agreements with the theoretical values. The echo power reference by applying Fast Fourier Transform (FFT) analysis has been used in calculating TS of the animal. The time domain of the echo evaluation in different angles shows the difference in the structure of the echo signal between the tortoise's body parts. This study reveals that high echo strength is acquired from the carapace and the plastron parts. The finding also showed that there are significant differences between $3,12,18$ years old turtles and fish in every angle measurement.

This is an open access article under the CC-BY-SA license.

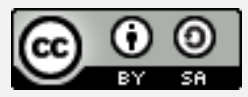

\section{Introduction}

Various technological applications and many of the new innovations in the field of acoustic and marine technology use sound. The sound is the pressure in the air or water medium of a physical wave whose speed depends on the movement of the vibration level through the medium. The movement of sounds faster in the water compared with the air is 1500 meters/second compared with 340 meters/second. Scientists believe that sounds are used by animals to communicate, attracting mate pairs, predatory alarms, and prey detection, as well as immigration navigation. In fish species, there are some capable of detecting chemical, mechanical, visual and electrical oils by peripheral sensor systems [1], in which the central nervous system interprets information and communicates physiologically for behavioral responses.

The decline of marine bird species globally has been linked to fishing operations that result in death. Some strategies can be undertaken to reduce incidental seabird catches such as the use of water cannons, acoustic barriers, magnetic deterrents and electrical barriers. The high frequency and emergency calls issued by commercial devices, the birds will experience temporary fear [2]. The catch rate can be reduced by acoustic devices without disrupting fishing activities by up to $50 \%$ compared to traditional monofilament networks. Traditional gillnets embedded with acoustic devices produce a sound signal 
capable of being heard by seabirds [3]. Incidental dolphin catching with nets can be avoided with gillnet acoustic devices (in Hector dolphin research) [4]. Hector's dolphin behavior can be observed for testing three devices (white, red and black acoustic devices) using in situ methods. Sound acoustic devices will be avoided by dolphin species, as well as bottlenose species. Bottlenose dolphins will avoid gillnet when acoustic devices emit sound [5], [6].

Troubleshooting marine animals unintentionally captured fishnets can be done using an effective tool with acoustic devices, which have been applied to whale species. The use of this device within 500 meters can reduce the number of dolphin species [7], as well as in the investigation that whale catches are obtained when added to the net, as they are trapped in the gillnets to zero. Sea turtles caught in fishing nets in the tropics associated with turtles trapped in the use of trawls are widely considered. This has an impact on the political and economic fields of fisheries and global commerce [8]. In fishing, a method that can be used to avoid accidentally caught turtles in fishnets can be done by dental modification, materials, and fishing methods [9]. The National Marine Fisheries Service (NMFS) provides a solution using a turtle tester (TED) on the use of larger shrimp prawns [10]. Separation of turtles and shrimp can be done with trawls since the 1980s [11]. Traditional TED consists of a metal network attached to an engraving net that can rescue sea turtles from online through trap doors [12].

The development of exclusion device technology is able to separate the animal lait on the network of fish and tortoise. The use of acoustic TEDs prevented turtles from entering fish tissue [13]. The device will transmit sound with 3 frequency bands between $200 \mathrm{~Hz}-15 \mathrm{kHz}$. This type of sound and frequency is capable of influencing the behavior of green turtles in sounds that have low-frequency modulation (LFM), so the animals will stay away when the sound is exposed to them [14]. Turtles are capable of being frightened using LFM sounds but only in one direction, and it is difficult to ensure the movement of the tortoise will approach or away from the net. Turtles must be detectable with the device so the sound repellents method is more efficient. The purpose of this research is to estimate the strength of reliable turtles. The design and improvement of Acoustic TED are done with the use of acoustic target strength (TS) technique to detect turtles and fish.

\section{Method}

\subsection{Aquatic animals target strength}

Acoustic technology in fishery applications has been done starting in the 1940s and after frequent and routine surveys on acoustics. Along with technological developments, the application of sonar is capable of providing solutions in significant aquatic acoustics [15]. Since the 1920s, the acoustic system most used in hydrographic and seabed mapping is echosounder [16]. Factors in acoustic methods such as acoustic size, target strength, or backscattering of individual cross-sections of organisms are used in estimating animal abundance in nature [17].

Marine animals body are complicated, a target strength (TS) can be effect through shape, size, orientation, swim bladder and so on [18]. Animals body size is an important factor in TS calculation, the size of the body affects the power of the echo, the greater the body has a higher echoing power [19], [20]. Morphological parameter of swimbladder, such as length and area can effect TS value [18]. Moreover, echo waveform from different tilt angles of swimbladder will contribute different TS value [21]. To obtain accurate TS value researcher has been used many approach to model animals body approximation, for example a fish body and swimbladder can be modeled as a series of finite cylinder and the total backscatter was estimated by summing the backscatter overall all the cylinders [21]-[23]. The variations of fish TS are influenced by natural variations of the volume and shape of the swim bladder.

Generally, most of the investigation TS of fish was focused on swimbladder. A study on TS two species of fish showed Gadolds swimbladder contribute $90 \%$ to $95 \%$ of backscattering cross section. Furthermore, the result also proved although species similar in size, shape and anatomy but each of fish contributed different TS value [24], [25]. The current measurement showed that acoustic research on species of capelin during the different season was found that TS was lower. One of the reason highlighted 
is capelin may having a higher fat content during autumn. The average TS can be reduced if there is a reduction in the size of the swimbladder as a result of fat content [26].

The TS value for mammals may depend on their air content in lung. A research conducted on two species of Whale found that Humpback whale and Right whale have different TS values. The possible reason is the presence of the air content in lung and suspected to be a major reflector [27]. Furthermore, Spinner dolphins have a combination of unique scattering characteristics to separate themselves from other animals. Their lungs produce strong echoes [28].

The other factor can affect the TS value of aquatic animals is orientation and angle of measurement. The incident signal perpendicular to the swim bladder resulted in the strongest TS [21], [29]. Studies on TS Myctophid species explain that the increase in fish TS relative to the roll angle of $5^{\circ}$ and $10^{\circ}$ on their dorsal aspect. The larger angle produces a larger target force, this raises the maximum change in the target power of $3 \mathrm{~dB}[30]$.

Study on TS of the aquatic shell animals are very limited and undiscovered. The first consideration is high TS values is contributed from it hard shell. The study suggests that shelled animal is somewhat difficult in obtaining acoustic strength due to several complicated factors such as body shape, biological properties, and acoustic scattering characteristics [22], [31]. A scattering from elastic shelled animal is depending on their hard shell [32]. Moreover, animal shells have significant meaning in scattering seafloor [29].

\subsection{Animal target strength measurement using reference target}

Several type of sphere have been used as calibration and reference target in determine animals TS, in particularly, ping pong ball [33], cooper, tungsten [34], [35] and steel ball [30], [36]. Generally, measurements and comparisons can be performed on envelope voltage target and the envelope voltage reference target (TS sphere). The standard target technique is both accurate and simple to apply in practice [37]. Acoustics is the most effective tool used to observe underwater objects, which are capable of transmitting long-distance sounds in water [38]. Underwater estimation process using echo-integrator method is done with sonar calibration result [39]. Known acoustic properties can facilitate the echosounder calibration process.

Consider a perfect sphere, not just a shape, but also immobile, rigid and very large compared to the Target power (TS) to return an echo that refers to a target's ability. The target could be a submarine, a mine, a whale, or a sinking ship. In sonar equations, the target strength is defined as $10 \log$ ratio of the acoustic intensity of the incident at the reflected acoustic intensity, referenced to the specific distance from the target acoustic center [40]. The definition of TS can be shown by computing the TS of a sphere, larger compared to a wavelength, on the assumption that the sphere is an isotropic reflector. The sphere is a perfect surface and reflected power uniformly in all directions, reflected intensity is given by (1).

$$
I r=\pi a^{2} \frac{I i}{4 \pi r^{2}}
$$

where $I r$ is the intensity of the reflection at range $\mathrm{r}, I i$ is the incident intensity and $a$ is the radius of sphere. At the reference distance of 1 yard, the reflected intensity ratio $I r$ to the incident intensity $I i$ is (2).

$$
\frac{I r}{I i}=\frac{a^{2}}{4}
$$

and the TS of the sphere becomes (3).

$$
\mathrm{TS}=10 \log \frac{I r}{I i}=10 \log \frac{a^{2}}{4}
$$

In practical work, spheres make good reference targets for sonar because their TS are relatively independent of orientation. The use of sphere as reference in calculating the value of the TS of animals 
is not new. The sphere has been use as reference target in calculating TS of clupeoids and gadoids species [41]. In addition, the same method has been used to study TS of krill [42] and TS of squid [36].

The target strength is largely measured by conventional methods, where peaks and mean intensities are calculated from irregular echo envelopes with 1-yard reduction of the length range that has been made [43]. By this method, reflected intensity from the object will be compared with the reflected intensity from the sphere as shown in Fig 1.

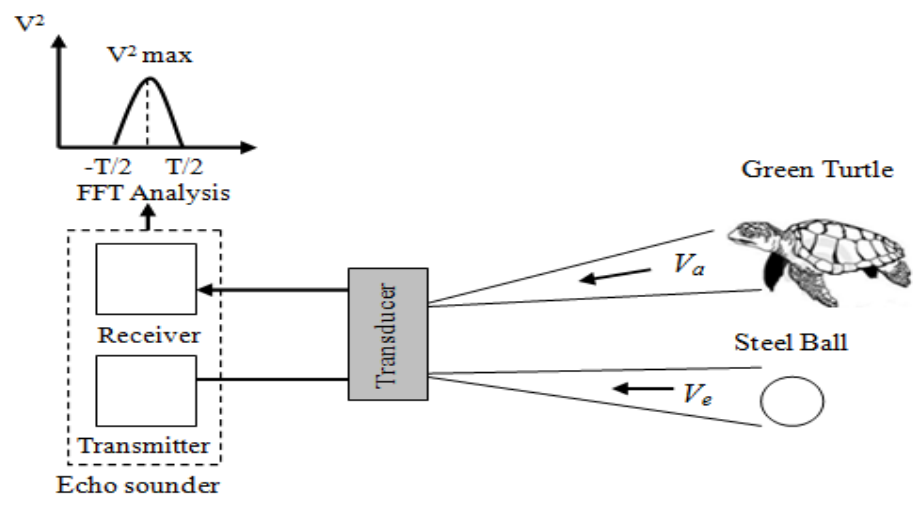

Fig. 1. Target strength measurements using a reference sphere

Based on Fig. 1, we can express TS by (4).

$$
\mathrm{TS}=10 \log +\frac{I_{1}}{I o_{\circ}}+T S r
$$

where $I_{1}$ is the echo intensity of object, $I_{o}$ is the echo intensity of the reference target (sphere), and TSr is the known target strength of the sphere from the (3). However, most of the target strength measurement of the intensity value is always referred as peak square [31]. The TS of the marine animals like fish, squids and shrimps can be represented by (5).

$$
\mathrm{TS}=10 \log +\frac{V o_{\mathrm{a}}{ }^{2}}{V_{\mathrm{e}} r^{2}}+T S r
$$

where $V_{0}^{2}$ is the voltage received by the echosounder from the animal and $V_{\mathrm{r}^{2}}$ is the voltage received from a sphere at the same range. Equation (5) involved envelope voltage, where the methods of measurement are done by substituting the steel ball with animals and compare the echo level. In the frequency domain the signal can be analyze as energy and power. Sometimes not all analysis can be done using time domain method, particularly when dealing with the thousand signals which has similar amplitude voltage and complicated shape. Therefore, power measurement in frequency domain using Fast Fourier Transform (FFT) analysis is another way to differentiate each of signals. The TS animal also can be obtained from power of FFT where can defined as (6).

$$
\mathrm{TS}=10 \log +\frac{\text { FFTanimals }}{\text { FFTsphere }}+\text { TSr }
$$

\subsection{Measurement procedure}

The research has been conducted at Turtle and Marine Ecosystem Centre (TUMEC), Rantau Abang, Dungun, Terengganu, Malaysia. The echoes of Green Turtle and fish have been recorded by using a dual frequency echosounder. Prior the measurement, there were several parameters that need to be set on the echosounder such as frequency, gain, noise reduction, echo level, STC/TVG, echo dynamic range and sound speed. The setting has been applied for all echoes recording measurement involved in this study.

Echosounder used in this study has a dual-mode frequency of 50 and $200 \mathrm{kHz}$. The frequency has been set operating at $200 \mathrm{kHz}$. Although, that frequency provide narrow beam but it can give higher resolution and have more precise data. In addition, by using this operating frequency the reflected signal from tank wall can be minimize. Echoes are shown using up to seven different colors. The colors 
currently used to show echoes are shown in the form of a color scale, the top color (originally blue) and the bottom color of the tip (initially red) represent the weakest and strongest echoes, respectively.

Gain of the echosounder output has been set at $35 \mathrm{~dB}$ which is at the medium rate. High gains setting will cause all weak echo exalted and will affect the measurement, especially for detecting the echo strength of fish. Meanwhile for low gain setting will cause all weak signal lost, especially for long distance measurement. The received noise level has been set at low level. Therefore, the received echosounder cleans the low noise echo signal. The animals involved in this study is Green Turtle and three species of fish as depicted in Table 1 and Table 2. The echo recording measurement has been conducted in static condition. There were five angle measurements of green turtles conducted in this study. The positions involved in this measurement were head, tail, side, carapace and plastron. This angle has been considered as the possible position of sea turtles direction during fishing trawl operation.

Table 1. Turtle's morphology

\begin{tabular}{cccccc}
\hline No & $\begin{array}{c}\text { Age } \\
\text { (year) }\end{array}$ & Sex & $\begin{array}{c}\text { Carapace } \\
\text { Length } \\
(\mathbf{c m})\end{array}$ & $\begin{array}{c}\text { Carapace } \\
\text { Wide } \\
(\mathbf{c m})\end{array}$ & $\begin{array}{c}\text { Weight } \\
\mathbf{( K g )}\end{array}$ \\
\hline 1. & 1 & Male & 25 & 23 & 1.8 \\
2. & 3 & Female & 35 & 31 & 3.5 \\
3. & 12 & Male & 61 & 56 & 27 \\
4. & 18 & Male & 72 & 61 & 60 \\
\hline
\end{tabular}

Table 2. Characterization of fish species

\begin{tabular}{cccc}
\hline No & Species & $\begin{array}{c}\text { Length } \\
(\mathbf{c m})\end{array}$ & $\begin{array}{c}\text { Width } \\
(\mathbf{c m})\end{array}$ \\
\hline 1. & Indian Scad & 19 & 4.5 \\
2. & Indian Mackerel & 18 & 4 \\
3. & Bigeye Scad & 21.5 & 5.2 \\
\hline
\end{tabular}

Prior the measurement, the sea turtle will attached to the wooden frame to ensure that the turtle position perpendicular to the sound beam. Other than that, it also can help to move the turtle to the other distances. The position of sea turtle, device connection and measurement distance illustrated in Fig. 2.

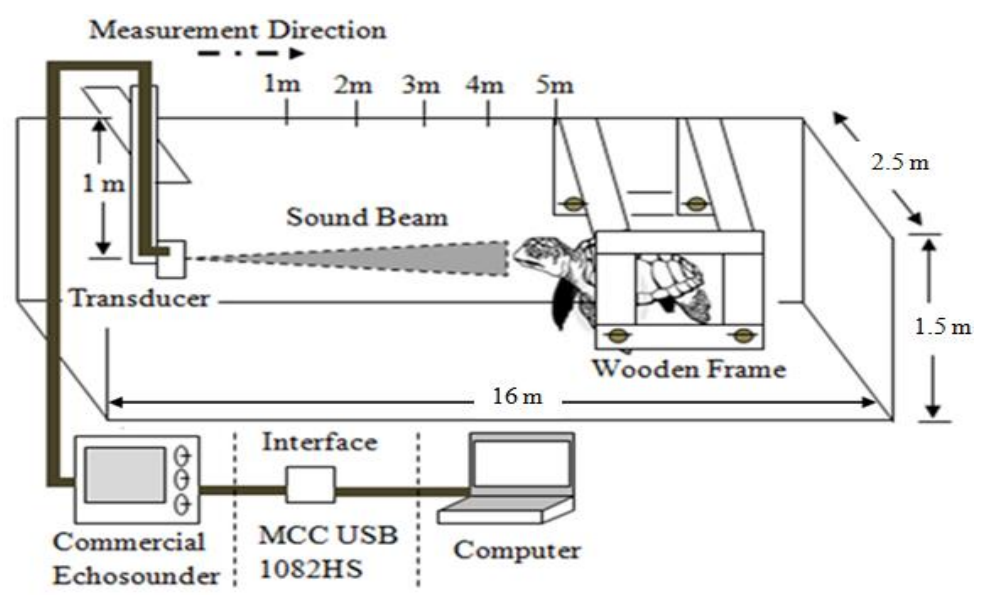

Fig. 2. The illustration of experiment setup

The echo signal has been recorded using an echo recording program which was created using MATLAB software. The method of recording echo signals from the turtle and fish was conducted by moving a wooden frame from 1 meter to 5 meters. By this method, the transducer is placed in a static condition to ensure the reference range for the echosounder is the same on all measurements. Measurements carried out on all five turtles body angle of the head, tail, side, carapace and plastron. A 
total of 45 samples of signal were recorded at every distance. The echo samples were saved as an Excel file in the laptop. The echo recording process is depicted in Fig. 3.

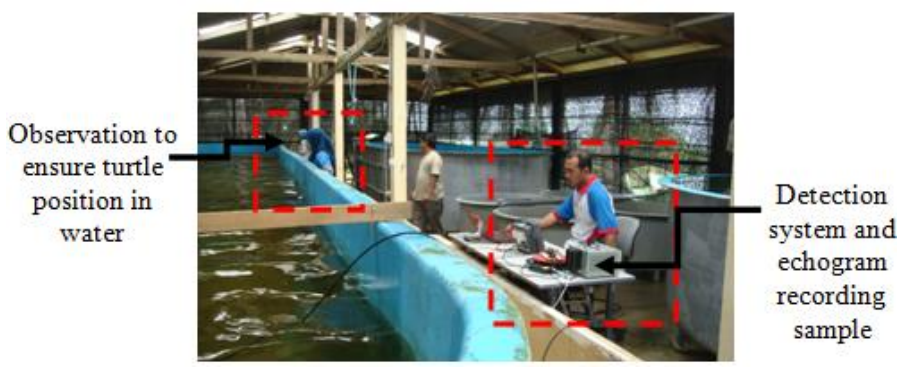

Fig. 3. The echo of animals recording process

\section{Results and Discussion}

The echo signal of green turtle and three species of fish has been recorded using MATLAB software and all sample data was saved in Microsoft Office Excel file. There are four green turtle involved in this experiment which are 1, 3,12 and 18 years old turtles. The observations focused on the head, tail, side, carapace and plastron angles for 1 meter to 5 meter distance. Meanwhile, there are three angles measurement involved for fish species which is head, tail and lateral angles. The echo measurement of turtle conducted in static condition and sample has been collected randomly from STC/TVG output for each distance.

The signal was sampled at $1 \mathrm{MHz}$ using multifunction DAQ device. This sampling rate, it is capable for recording the amplitude of the sound as fast as possible and can represent actual signal [21]. Fig. 4 demonstrated the echo signal of 1 year Green Turtle at 5 meters distances. Based on the result, it obviously showed signal pattern are different for each angles. The highest amplitude is obtained from carapace and plastron parts. The head, tail and side of sea turtle showed have similar amplitude but different pattern of signal where each angles consist double signal.
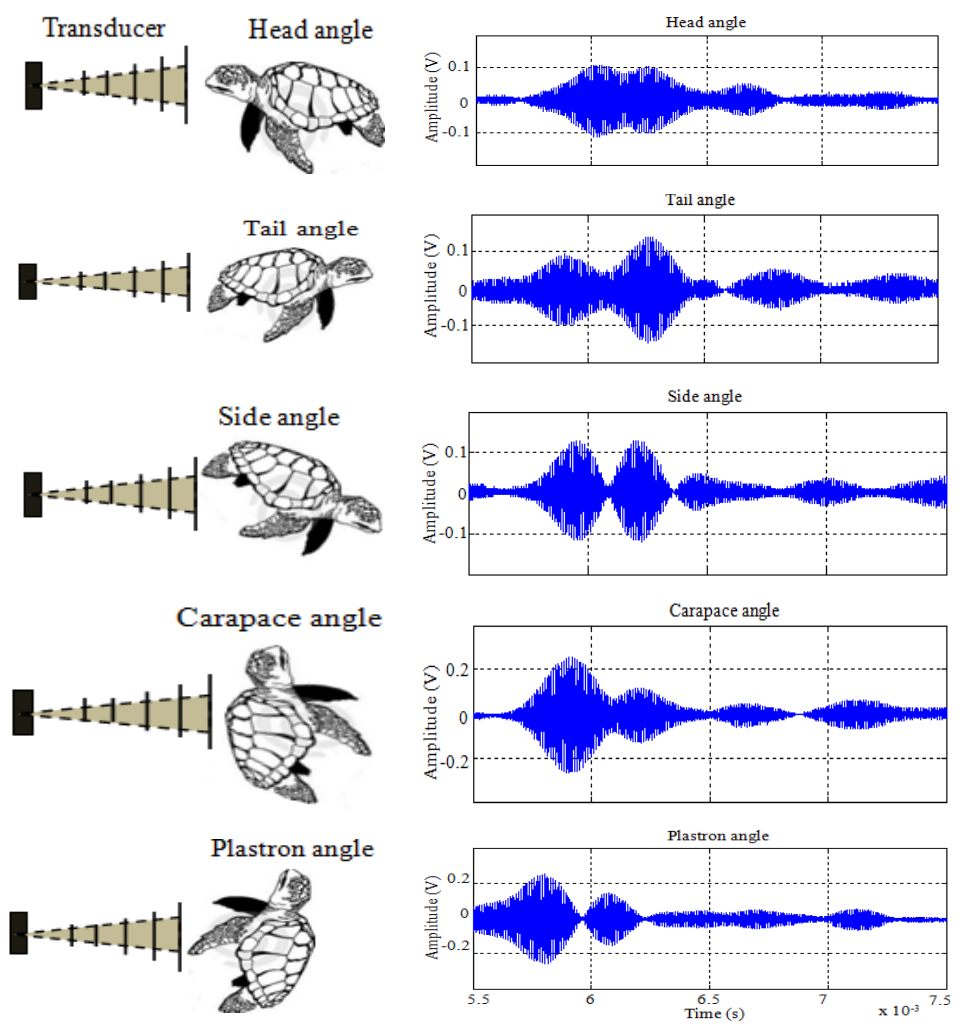

Fig. 4. Reflected echo signal of one year Green Turtle at different angles 
The envelope echoes from the three species of fish namely Indian Mackerel, Indian Scad, and Bigeye Scad have been successfully recorded. The experiment procedure is same with the green turtle where the echo signal has been recorded at 1 to 5 meters distances. The observations focused on the head, tail, and lateral side. The echo recording program of Matlab software is used to collect samples randomly. The example envelope echo of Indian Mackerel at 5 meters distance is shown in Fig. 5.
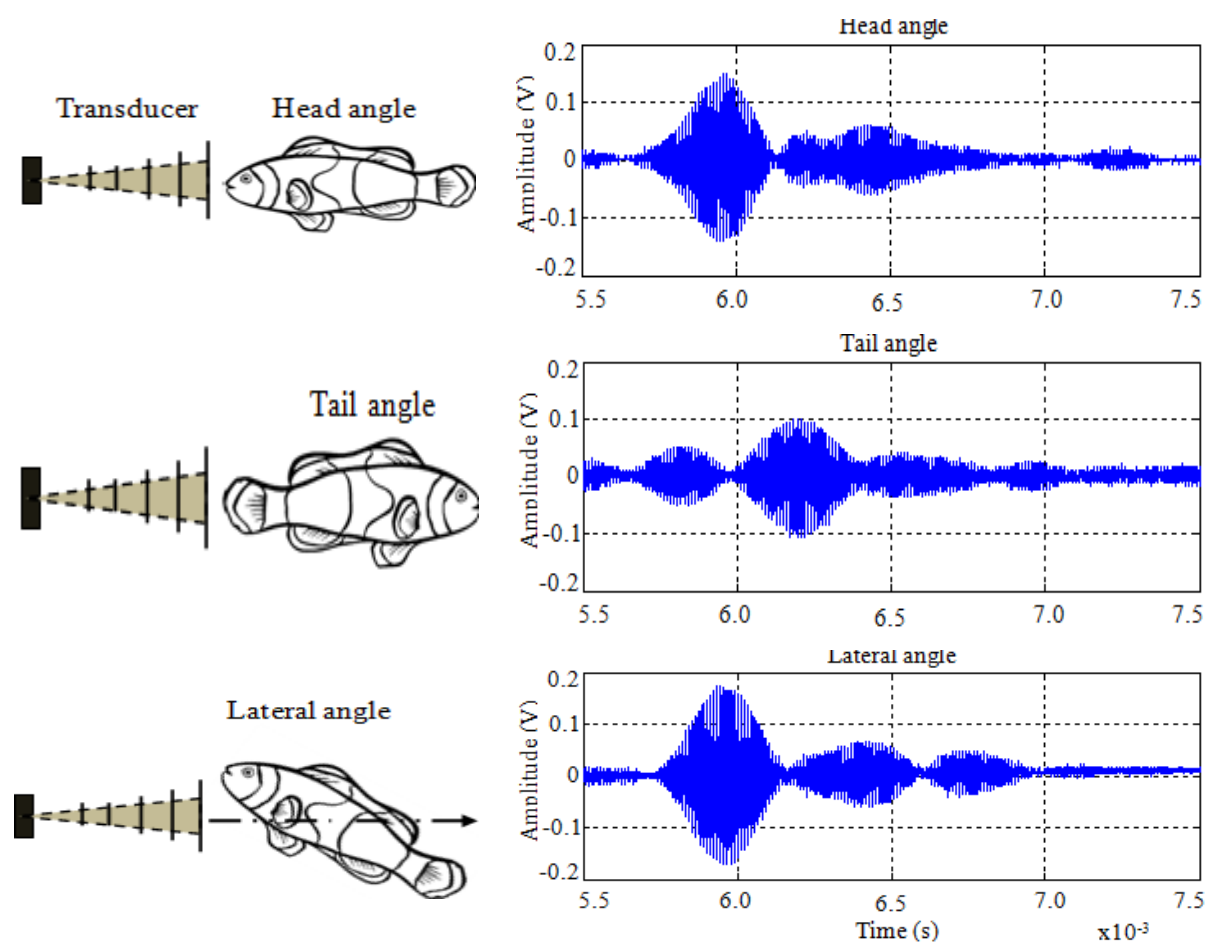

Fig. 5. Reflected echo signal of Indian Mackerel at different angles

Based on the result discovered that envelope echo for sea turtle and fish have different signal pattern and amplitude. The signal of sea turtle and fish then was conducted in FFT analysis. The total FFT magnitude of animals has been recorded and compared with the reference target.

In this study, $0.0215 \mathrm{~m}$ radius of the steel ball was used as a reference target. Reflected value from turtle and fish was referring to reflected value from steel ball for each distance. TS of the steel ball were calculated by (3), suggested by Urick [43], which is equal to $-39.372 \mathrm{~dB}$. The TS average of the green turtle and fish from 1 to 5 meters have been compared as shown in Fig. 6 . The TS was calculated using echo power reference method which involved FFT analysis. Based on the observation, the highest TS value of fish was from lateral part which is recorded for all species in range -38.282 to $-38.110 \mathrm{~dB}$. Meanwhile the lowest value recorded was from Indian Scad at tail angle is $-39.546 \mathrm{~dB}$.

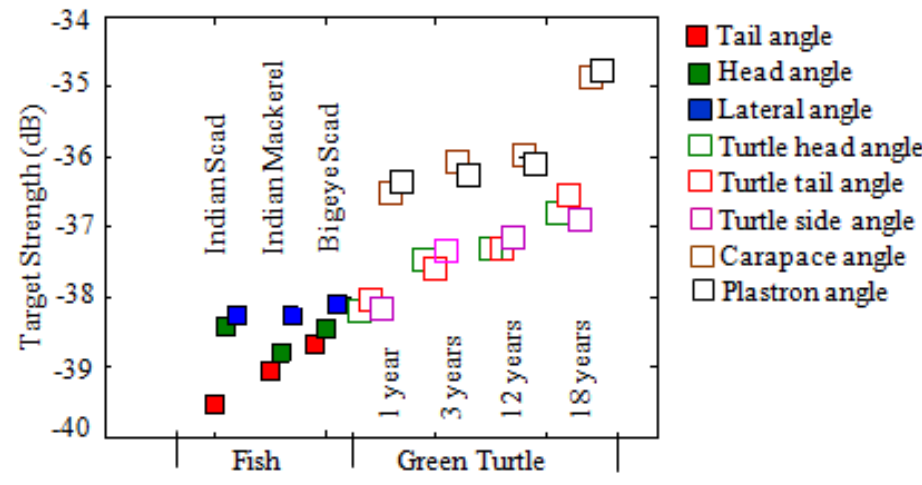

Fig. 6. Average TS of Green Turtle and fish 
The analysis of Green Turtle TS showed that the two highest value is from 18 years old turtle at carapace and plastron angle. The TS value range recorded for all aged of turtle at carapace angle is -6.514 to $-34.871 \mathrm{~dB}$ and at plastron angle is -36.371 to $-34.769 \mathrm{~dB}$. The scatter graph indicates that there are significant different between turtle aged 3,12, 18 years old and fish at all angle measurement. However, there are no significant different between turtle age 1 year old and fish at head, tail and side angle.

\section{Conclusion}

The echo signal of Green Turtle and three species of fish was measured at TUMEC, Rantau Abang, Terengganu, Malaysia. The experiment were conducted in a research fiber tank using an echosounder. The target strength (TS) of animals was obtained using echo power value which involved steel ball as reference target. The echo power reference by applying FFT can facilitate in calculating TS of the animal, particularly involve complicated structural of echo signal. The comparison TS value between Green Turtle and fish showed there are significant different for 3,12 and 18 years Green Turtle. In addition, this study also reveals that high TS is acquired from the carapace and the plastron parts. This result also shows that the determination of TS tortoises is based on several factors such as size, surface, and angle. Based on the results, the conclusion is obtained that the adult Green Turtles can be distinguished by the three fish samples used by the methods undertaken in this study. Although the study shows significant findings, further research should be conducted for other fish species and TS values of turtles and fish are indispensable. This study has limited distance and space as it is done in fiber tanks, so further research is recommended to be done in large ponds and open ocean.

\section{Acknowledgment}

We thank the TUMEC staff for expert sharing, especially in handling the turtles and providing facilities during the experiments. We also want to thank those who were involved in the experiments, directly or indirectly, to ensure the success of this study. We likewise thank to Department of Electrical Engineering, Politeknik Sultan Hj Ahmad Shah and Faculty of Electrical \& Electronics Engineering, Universiti Malaysia Pahang, for providing all the facilities and encouraging a culture of research and innovation among students.

\section{References}

[1] L. K. Jordan, J. W. Mandelman, D. M. McComb, S. V. Fordham, J. K. Carlson, and T. B. Werner, "Linking sensory biology and fisheries bycatch reduction in elasmobranch fishes: a review with new directions for research," Conserv. Physiol., vol. 1, no. 1, p. $\cot 002-\cot 002$, Apr. 2013, doi: https://doi.org/10.1093/conphys/cot002.

[2] L. Bull, A review of methodologies for mitigating incidental catch of seabirds in New Zealand fisheries. Science \& Technical Pub., Department of Conservation, 2007, available at: https://www.doc.govt.nz/Documents/science-and-technical/drds263.pdf.

[3] S. Løkkeborg, "Best practices to mitigate seabird bycatch in longline, trawl and gillnet fisheries-efficiency and practical applicability," Mar. Ecol. Prog. Ser., vol. 435, pp. 285-303, Aug. 2011, doi: https://doi.org/10.3354/meps09227.

[4] G. S. Stone, L. Cavagnaro, A. Hutt, S. Kraus, K. Baldwin, and J. Brown, "Reactions of Hector's dolphins to acoustic gillnet pingers," Conserv. Serv. Levy Contract Rep., vol. 3071, 2000, available at: http://nationalparks.co.nz/Documents/science-and-technical/CSL3071.PDF.

[5] T. M. Cox, A. J. Read, D. Swanner, K. Urian, and D. Waples, "Behavioral responses of bottlenose dolphins, Tursiops truncatus, to gillnets and acoustic alarms," Biol. Conserv., vol. 115, no. 2, pp. 203-212, Feb. 2004, doi: https://doi.org/10.1016/S0006-3207(03)00108-3.

[6] M. Gazo, J. Gonzalvo, and A. Aguilar, "Pingers as deterrents of bottlenose dolphins interacting with trammel nets," Fish. Res., vol. 92, no. 1, pp. 70-75, Jul. 2008, doi: https://doi.org/10.1016/j.fishres.2007.12.016.

[7] P. Berggren, J. Carlström, and N. Tregenza, "Mitigation of small cetacean bycatch; evaluation of acoustic alarms (MISNET)," Rep Int Whal Comm SC/54/SM2, pp. 1-28, 2002. 
[8] J. W. Valdemarsen and P. Suuronen, "19 Modifying Fishing Gear to Achieve Ecosystem Objectives," Responsible Fish. Mar. Ecosyst., p. 321, 2003.

[9] E. Gilman et al., "Mitigating sea turtle by-catch in coastal passive net fisheries," Fish Fish., vol. 11, no. 1, pp. 57-88, Mar. 2010, doi: https://doi.org/10.1111/j.1467-2979.2009.00342.x.

[10] R. L. Lewison, L. B. Crowder, and D. J. Shaver, "The Impact of Turtle Excluder Devices and Fisheries Closures on Loggerhead and Kemp's Ridley Strandings in the Western Gulf of Mexico," Conserv. Biol., vol. 17, no. 4, pp. 1089-1097, Aug. 2003, doi: https://doi.org/10.1046/j.1523-1739.2003.02057.x.

[11] S. P. Epperly, "Fisheries-Related Mortality and Turtle Excluder Devices (TEDs)," in The Biology of Sea Turtles, Volume II, P. L. Lutz, J. A. Musick, and J. Wyneken, Eds. New York, New York, USA: CRC Press, 2002, p. 339.

[12] B. J. Gallaway, J. G. Cole, J. M. Nance, R. A. Hart, and G. L. Graham, "Shrimp Loss Associated with Turtle Excluder Devices: Are the Historical Estimates Statistically Biased," North Am. J. Fish. Manag., vol. 28, no. 1, pp. 203-211, Feb. 2008, doi: https://doi.org/10.1577/M07-002.1.

[13] M. Lenhardt, "Sea turtle auditory behavior," J. Acoust. Soc. Am., vol. 112, no. 5, pp. 2314-2314, Nov. 2002, doi: https://doi.org/10.1121/1.1526585.

[14] A. Yudhana, "Turtle Hearing Classification for Turtle Excluder Devices Design," Universiti Teknologi Malaysia, 2011.

[15] D.-Z. Chu, "Technology evolution and advances in fisheries acoustics," J. Mar. Sci. Technol., vol. 19, no. 3, pp. 245-252, 2011, available at: http://www.airitilibrary.com/Publication/PublicationIndex/10232796.

[16] X. Lurton and S. DeRuiter, "Sound radiation of seafloor-mapping echosounders in the water column, in relation to the risks posed to marine mammals," Int. Hydrogr. Rev., no. 6, 2011, available at: https://journals.lib.unb.ca/index.php/ihr/article/view/20886.

[17] D. N. MacLennan and E. J. Simmonds, Fisheries acoustics, 1st ed., vol. 5. Springer Netherlands, 1992, available at: https://www.springer.com/gp/book/9780412330605.

[18] K. Abe, K. Sadayasu, K. Sawada, K. Ishii, and Y. Takao, "Precise target strength measurement and morphological observation of juvenile walleye pollock (Theragra chalcogramma)," in Oceans '04 MTS/IEEE Techno-Ocean '04 (IEEE Cat. No.04CH37600), 2004, vol. 1, pp. 370-374, doi: https://doi.org/10.1109/OCEANS.2004.1402945.

[19] K. J. Benoit-Bird and W. W. L. Au, "Echo strength and density structure of Hawaiian mesopelagic boundary community patches," J. Acoust. Soc. Am., vol. 114, no. 4, pp. 1888-1897, Oct. 2003, doi: https://doi.org/10.1121/1.1612484.

[20] J. Frouzova and J. Kubecka, "Changes of acoustic target strength during juvenile perch development," Fish. Res., vol. 66, no. 2-3, pp. 355-361, Feb. 2004, doi: https://doi.org/10.1016/S0165-7836(03)00182-6.

[21] K. J. Benoit-Bird, W. W. L. Au, and C. D. Kelley, "Acoustic backscattering by Hawaiian lutjanid snappers. I. Target strength and swimbladder characteristics," J. Acoust. Soc. Am., vol. 114, no. 5, p. 2757, 2003, doi: https://doi.org/10.1121/1.1614256.

[22] T. Stanton, "Review and recommendations for the modelling of acoustic scattering by fluid-like elongated zooplankton: euphausiids and copepods," ICES J. Mar. Sci., vol. 57, no. 4, pp. 793-807, Aug. 2000, doi: https://doi.org/10.1006/jmsc.1999.0517.

[23] K. Amakasu and M. Furusawa, "Effective frequency for acoustic survey of antarctic krill," in Oceans '04 MTS/IEEE Techno-Ocean '04 (IEEE Cat. No.04CH37600), 2004, vol. 1, pp. 375-382, doi: https://doi.org/10.1109/OCEANS.2004.1402946.

[24] K. G. Foote, "Importance of the swimbladder in acoustic scattering by fish: A comparison of gadoid and mackerel target strengths," J. Acoust. Soc. Am., vol. 67, no. 6, pp. 2084-2089, Jun. 1980, doi: https://doi.org/10.1121/1.384452.

[25] S. Sunardi, A. Yudhana, J. Din, and R. B. R. Hassan, "Swimbladder on fish target strength," TELKOMNIKA (Telecommunication Comput. Electron. Control., vol. 6, no. 2, p. 139, Aug. 2008, doi: https://doi.org/10.12928/telkomnika.v6i2.561. 
[26] R. Jørgensen, "Acoustic target strength of capelin measured by single-target tracking in a controlled cage experiment," ICES J. Mar. Sci., vol. 59, no. 5, pp. 1081-1085, Oct. 2002, doi: https://doi.org/10.1006/jmsc.2002.1239.

[27] J. H. Miller and D. C. Potter, "Active high frequency phased-array sonar for whale shipstrike avoidance: target strength measurements," in MTS/IEEE Oceans 2001. An Ocean Odyssey. Conference Proceedings (IEEE Cat. No.01CH37295), 2001, vol. 4, pp. 2104-2107, doi: https://doi.org/10.1109/OCEANS.2001.968324.

[28] K. J. Benoit-Bird and W. W. L. Au, "Prey dynamics affect foraging by a pelagic predator (Stenella longirostris) over a range of spatial and temporal scales," Bebav. Ecol. Sociobiol., vol. 53, no. 6, pp. 364-373, 2003, available at: https://link.springer.com/article/10.1007/s00265-003-0585-4.

[29] T. K. Stanton, "On acoustic scattering by a shell-covered seafloor," J. Acoust. Soc. Am., vol. 108, no. 2, pp. 551-555, Aug. 2000, doi: https://doi.org/10.1121/1.429585.

[30] K. J. Benoit-Bird and W. W. L. Au, "Target strength measurements of Hawaiian mesopelagic boundary community animals," J. Acoust. Soc. Am., vol. 110, no. 2, pp. 812-819, Aug. 2001, doi: https://doi.org/10.1121/1.1382620.

[31] T. Mukai, K. Iida, Y. Ando, H. Mikami, Y. Maki, and R. Matsukura, "Measurements of swimming angles, density, and sound speed of the krill euphausia pacifica for target strength estimation," in Oceans '04 MTS/IEEE Techno-Ocean '04 (IEEE Cat. No.04CH37600), 2004, vol. 1, pp. 383-388, doi: https://doi.org/10.1109/OCEANS.2004.1402947.

[32] J. D. Warren, T. K. Stanton, D. E. McGehee, and Dezhang Chu, "Effect of animal orientation on acoustic estimates of zooplankton properties," IEEE J. Ocean. Eng., vol. 27, no. 1, pp. 130-138, 2002, doi: https://doi.org/10.1109/48.989899.

[33] A. Drew, "Initial Results from a Portable Dual-Beam Sounder for In Situ Measurements of Target Strength of Fish," in OCEANS '80, 1980, pp. 376-380, doi: https://doi.org/10.1109/OCEANS.1980.1151378.

[34] J. M. Jech, D. Chu, K. G. Foote, T. R. Hammar, and L. C. Huffnagle, "Calibrating two scientific echo sounders," in Oceans 2003. Celebrating the Past ... Teaming Toward the Future (IEEE Cat. No.03CH37492), 2003, p. 1625-1629 Vol.3, doi: https://doi.org/10.1109/OCEANS.2003.178117.

[35] K. G. Foote and D. N. MacLennan, "Comparison of copper and tungsten carbide calibration spheres," $J$. Acoust. Soc. Am., vol. 75, no. 2, pp. 612-616, Feb. 1984, doi: https://doi.org/10.1121/1.390489.

[36] I. N. Arnaya, N. Sano, and K. IIDA, "Studies on Acoustic Target Strength of Squid: I . Intensity and energy target strengths," Bull. Fac. Fish. Hokkaido Univ., vol. 39, no. 3, pp. 187-200, 1988, available at: https://eprints.lib.hokudai.ac.jp/dspace/bitstream/2115/24002/1/39(3)_P187-200.pdf.

[37] J. Simmonds and D. MacLennan, Eds., Fisheries Acoustics: Theory and Practice, 2005, doi: https://doi.org/10.1002/9780470995303.

[38] S. Sarangapani, J. H. Miller, G. R. Potty, D. B. Reeder, T. K. Stanton, and Dezhang Chu, "Measurements and modeling of the target strength of divers," in Europe Oceans 2005, 2005, p. 952-956 Vol. 2, doi: https://doi.org/10.1109/OCEANSE.2005.1513185.

[39] D. N. MacLennan, "The Theory of Solid Spheres as Sonar Calibratlcm Targets," Scott. Fish. Res. Rep, 1981, available at: http://www.gov.scot/Uploads/Documents/SFRR22.pdf.

[40] R. P. Hodges, Underwater Acoustics: Analysis, Design and Performance of Sonar, 2010, doi: https://doi.org/10.1002/9780470665244.

[41] O. Nakken and K. Olsen, "Target strength measurements of fish," Rapp. Procès-Verbaux des Réunions Cons. Int. pour l'Exploration la Mer, vol. 170, pp. 52-69, 1977, available at: http://hdl.handle.net/11250/107967.

[42] J. Kalinowski, A. Dyka, and L. Kilian, "Target strength of krill," Pol. Polar Res, vol. 1, no. 4, pp. 147-153, 1980, available at: http://polish.polar.pan.pl/ppr01/1980_4_147-153.pdf.

[43] R. J. Urick, Principles of underwater sound for engineers, 3rd ed. McGraw-Hill, 1983. 